GT2020-14398

\title{
UNSTEADY SIMULATIONS OF A TRAILING-EDGE SLOT USING MACHINE-LEARNT TURBULENCE STRESS AND HEAT-FLUX CLOSURES
}

\author{
Chitrarth Lav; Richard D. Sandberg \\ Department of Mechanical Engineering \\ University of Melbourne, Australia \\ Email: chitrarth.lav@unimelb.edu.au
}

\section{ABSTRACT}

The trailing edge slot is a canonical representation of the pressure-side bleed flow encountered in high pressure turbines. Predicting the flow and temperature downstream of the slot exit remains challenging for RANS and URANS, with both significantly overpredicting the adiabatic wall-effectiveness. This overprediction is attributable to the incorrect mixing prediction in cases where the vortex shedding is present. In case of RANS the modelling error is rooted in not properly accounting for the shedding scales while in URANS the closures account for the shedding scales twice, once by resolving the shedding and twice with the model for all the scales. Here, we present an approach which models only the stochastic scales that contribute to turbulence while resolving the scales that do not, i.e. scales considered as contributing to deterministic unsteadiness. The model for the stochastic scales is obtained through a data-driven machine learning algorithm, which produces a bespoke turbulence closure model from a high-fidelity dataset. We use the best closure (blowing ratio of 1.26) for the anisotropy obtained in the a priori study of Lav, Philip \& Sandberg [A New Data-Driven Turbulence Model Framework for Unsteady Flows Applied to Wall-Jet and Wall-Wake Flows, 2019] and conduct compressible URANS calculations. In the first stage, the energy equation is solved utilising the standard gradient diffusion hypothesis for the heat-flux closure. In the second stage, we develop a bespoke heat-flux closure using the machine-learning approach for the stochastic heat-flux components only. Subsequently, calculations are performed using the machine-learnt closures for the heat-flux and the anisotropy together. Finally, the generalisability of the developed closures is evaluated by testing them on additional blowing ratios of 0.86 and 1.07. The machine-learnt closures developed specifically for URANS calculations show significantly improved predictions for the adiabatic wall-effectiveness across the different cases.

*Address all correspondence to this author.

\author{
NOMENCLATURE \\ $\alpha, \alpha_{t} \quad$ Molecular and turbulent thermal diffusivities \\ $a_{i j}^{r e f} \quad$ Anisotropy tensor $=\tau_{i j} / 2 \rho k-\delta_{i j} / 3$ \\ $a_{i j}^{\text {mod }} \quad$ Model for the anisotropy tensor \\ $\eta \quad$ Adiabatic wall effectiveness $=\left(T_{w}-T_{f s}\right) /\left(T_{s}-T_{f_{s}}\right)$ \\ $I_{m}, J_{n} \quad$ Scalar invariants $\forall m \in[1,2]$ and $\forall n \in[1,5]$ \\ $k, \omega \quad$ Turbulence kinetic energy \& specific dissipation rate \\ $\Phi \quad$ Instantaneous value of a variable $\Phi=\bar{\Phi}+\Phi^{\prime}$ \\ $\bar{\Phi} \quad$ Time independent mean value of a variable $\Phi$ \\ $\Phi^{\prime} \quad$ Instantaneous unsteadiness of a variable $\Phi, \widetilde{\Phi}+\Phi^{\prime \prime}$ \\ $\widetilde{\Phi} \quad$ Deterministic contribution of $\Phi$ \\ $\Phi^{\prime \prime} \quad$ Stochastic contribution of $\Phi$ \\ $\mu, \mu_{t} \quad$ Molecular and turbulent viscosities \\ $\rho \quad$ Density of fluid \\ $\rho_{R S} \quad$ Alignment \\ $\operatorname{Pr}, \operatorname{Pr}_{t} \quad$ Molecular and turbulent Prandlt numbers \\ $S_{i j} \quad$ Mean strain rate tensor $=0.5\left(\partial u_{i} / \partial x_{j}+\partial u_{j} / \partial x_{i}\right)$ \\ $\Omega_{i j} \quad$ Mean rotation rate tensor $=0.5\left(\partial u_{i} / \partial x_{j}-\partial u_{j} / \partial x_{i}\right)$ \\ $s, t \quad$ Height of slot and thickness of lip \\ $\tau_{i j} \quad$ Reynolds stress tensor $=\overline{u_{i}^{\prime} u_{j}^{\prime}}$ \\ $T_{f s}, T_{s}, T_{w} \quad$ Temperature in freestream, slot and at the wall \\ $u_{i} \quad$ Velocity component in the $i$ th direction $\forall i \in[1,3]$ \\ $V_{i j}^{n} \quad$ Tensor basis functions $\forall n \in[1,3]$ \\ BR Blowing Ratio \\ DES Detached Eddy Simulations \\ GEP Gene-Expression Programming \\ LES Large Eddy Simulations \\ RANS Reynolds-Averaged Navier Stokes \\ URANS Unsteady Reynolds-Averaged Navier Stokes
}

\section{INTRODUCTION}

The trailing-edge slot is a canonical configuration representing the pressure-side bleed, i.e. the slots cut into the trailingedge of a high-pressure turbine. The purpose of these slots is to inject coolant to protect the surface of the blade from the hot working fluid, which drives the turbine, since the temperature of 
the working fluid can be much higher than the melting point of the blade material. This configuration has been studied before, both experimentally $[1,2]$ and numerically $[3,4]$, with the focus on understanding how effective the slot fluid is in shielding the blade surface, quantified by a parameter called the adiabatic wall-effectiveness $\eta$ :

$$
\eta=\frac{T_{w}-T_{f s}}{T_{s}-T_{f s}},
$$

where $T_{w}, T_{f s}$ and $T_{s}$ are the adiabatic wall, freestream and slot fluid temperatures respectively. Large $\eta$ indicates lower wall temperatures and vice versa. Numerically, this problem has been addressed using Reynolds-Averaged Navier-Stokes (RANS) [5, 6], unsteady RANS [4, 7], Detached Eddy Simulations (DES) [3,8] and Large Eddy Simulations (LES) $[4,9,10]$. From an industrial design perspective however, the latter two approaches remain prohibitively expensive for parametric studies and thus not suitable beyond providing understanding of the flow physics. While most of these previous works were devoted to designing effective cutback slots by studying the effect of varying the geometry, thickness of the slot and/or the coolant ejection speed, the fundamental problem of accurately predicting $\eta$ with the low-fidelity tools such as RANS and URANS still persists. The results from RANS have consistently demonstrated a significant overprediction of $\eta$ when compared with the highfidelity trends which has led to the now well-established understanding [3] that the trailing-edge slot exhibits strong unsteadiness in the form of vortex shedding. The effect of the shedding is an increase in the momentum mixing in the near slot exit that is responsible for the steep drop in $\eta$, which RANS could never predict, suggesting the need for using URANS instead. However, the results have not been encouraging, possibly being best highlighted by the study of Ivanova \& Laskowski [4], where they compared the performance of URANS alongside DES and LES. The overprediction in $\eta$ existed even though the vortex shedding was being resolved.

This poor prediction from both RANS and URANS has pushed for the need to build better models, which has seen a recent resurgence with the incorporation of machine-learning for model development. Specifically for the trailing-edge slot configuration, research by Sandberg et al. [10] and Milani et al. [11] is notable for demonstrating improvements using machine-learnt models. While the former study utilised a symbolic regression algorithm called Gene-Expression Programming (GEP) [12], the latter study utilised a random forest algorithm; both targeting the heat-flux closure when solving the scalar transport equation for temperature. This study, however, makes the distinction that the temperature distribution is strongly influenced by the momentum mixing as documented in other studies, and so builds a closure model not just for the heat-flux but also for the turbulence stress. As considered by Sandberg et al. [10], this study uses GEP as well, because it produces a tangible equation for the closure that can be implemented easily and requires high-fidelity data only during the closure development phase, not during the runtime, thus being computationally much cheaper than a neural network [13]. So, in this paper, we conduct unsteady simulations using new closures for the turbulence stress and the heat-flux obtained from the GEP machine-learning algorithm. Essentially,

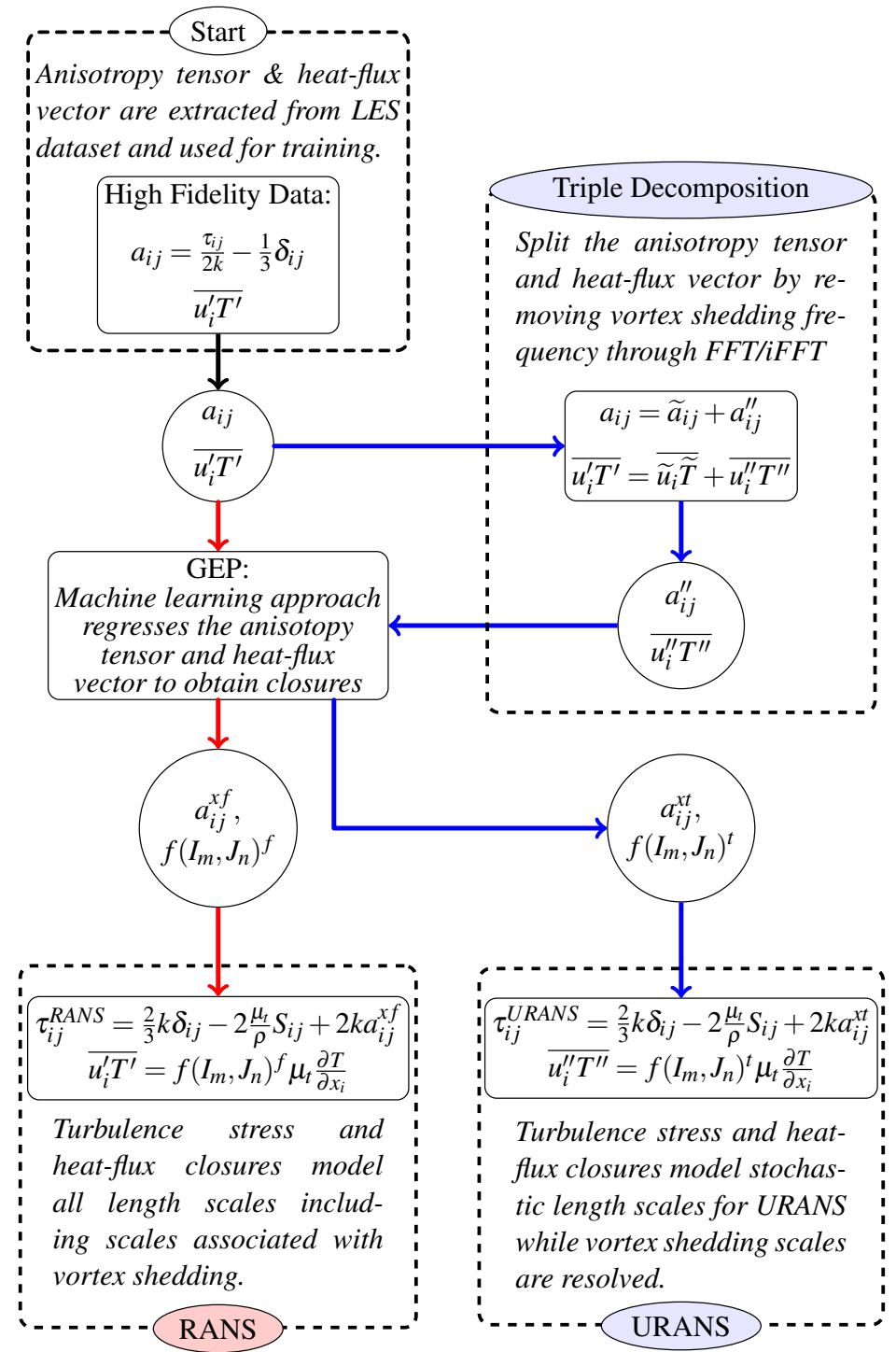

FIGURE 1: Machine-learning framework, adapted from Lav et al. [14] and modified to add the heat-flux vector processing.

this study is a direct extension of Lav et al. [14], where turbulence stress closures were developed for 3 blowing ratios (BR, defined later) and the best closure across all the BRs was suggested based on an a priori metric, i.e. without conducting any calculations. In this paper we, for the first time, evaluate the performance of the suggested closure for $\mathrm{BR}=1.26$ in an a posteriori sense, i.e. by conducting unsteady calculations. Secondly, we extend the framework of Lav et al. [15] from the turbulence stress closure development to include the decomposition of the turbulent heat-flux and the subsequent developement of a heatflux closure, testing it for the BR $=1.26$ case. Subsequently, we evaluate the performance of the turbulence stress and heat-flux closure by conducting coupled unsteady calculations. Finally, to evaluate the generalisability, the closures for $\mathrm{BR}=1.26$ case are simultaneously tested on additional BRs of 0.86 and 1.07 .

\section{MACHINE-LEARNING FRAMEWORK}

A closure for the turbulence stress ubiquitously used is the Boussinesq approximation, where the anisotropy tensor is mod- 
elled by the mean strain rate tensor $S_{i j}$

$$
a_{i j}^{\text {mod }}=-\frac{\mu_{t}}{\rho k} S_{i j}=-\frac{S_{i j}}{\omega} .
$$

Here $\rho, k, \omega$ and $\mu_{t}$ are the fluid density, turbulence kinetic energy, specific dissipation rate and eddy viscosity respectively. For the $k-\omega$ SST model [16], the relation between $\rho, k, \omega$ and $\mu_{t}$ above holds true. This model attempts to account for the actual anisotropy $a_{i j}^{r e f}=\tau_{i j} / 2 \rho k-\delta_{i j} / 3\left(\tau_{i j}=\overline{u_{i}^{\prime} u_{j}^{\prime}}\right.$ being the Reynolds stress tensor) and is responsible for poor prediction in RANS for various flow configurations [17]. In a URANS sense, it also results in accounting for the unsteady length scales, i.e. vortex shedding scales twice, once by resolving those scales and again when it models all length scales. So, using GEP, we build a better model for the anisotropy tensor that is more representative of the flow while also accounting for all scales but the vortex shedding scales. To make the distinction clearer, we refer to the vortex shedding scales as deterministic scales from now on, since they can be determined based on the shedding frequency and term the remaining as stochastic scales. For building an improved closure, it must be realised that the Boussinesq approximation is a linear closure, as $S_{i j}$ is directly proportional to $a_{i j}^{\text {mod }}$ and thus, improvements can be sought by considering a class of non-linear closures such as the Explict Algebraic Reynolds Stress Models (EARSMs) [18, 19] instead. These closures are composed of multiple tensor basis functions $\left(V_{i j}^{m}, \forall m \in[1,3]\right)$ and scalar invariants $\left(I_{m}, \forall m \in[1,2]\right)$

$$
\begin{aligned}
V_{i j}^{1} & =\frac{S_{i j}}{\omega}, \quad I_{1}=S_{m n} S_{n m}, \quad I_{2}=\Omega_{m n} \Omega_{n m}, \\
V_{i j}^{2} & =\frac{S_{i k} \Omega_{k j}-\Omega_{i k} S_{k j}}{\omega}, \\
V_{i j}^{3} & =\frac{S_{i k} S_{k j}-\frac{1}{3} \delta_{i j} S_{m n} S_{n m}}{\omega},
\end{aligned}
$$

where, $\Omega_{i j}$ is the mean rotation rate tensor. Using the framework developed in Lav et al. [15], the high-fidelity data is first processed to remove the deterministic scale contribution, followed by developing a non-linear closure for the stochastic scales, i.e. $a_{i j}^{\text {mod }}=f\left(V_{i j}^{1}, V_{i j}^{3}, V_{i j}^{3}, I_{1}, I_{2}\right)$. The processing of the high-fidelity data involves constructing the deterministic Reynolds stress tensor by keeping only the deterministic contribution from the highfidelity time series data. This is achieved by evaluating the vortex shedding frequency and then removing the remaining frequency information from the time series through Fast Fourier Transforms. For the trailing-edge slot configuration considered in this study, the high-fidelity data processing and closure development was performed in Lav et al. [14] for three blowing ratios (BR = $0.86,1.07$ and 1.26) with the suggestion of using the closure of $\mathrm{BR}=1.26$ on all the cases considered, based on an a priori assessment. All terms in Eqn. (3) were obtained from the LES, with $\omega$ obtained by solving the $\omega$ equation in the $k-\omega$ SST solver by freezing the velocity and turbulence kinetic energy fields.

Following the same process as for the turbulence stress closure development, a closure for the heat-flux can also be developed. The most commonly used closure for the heat-flux is the standard gradient diffusion hypothesis (SGDH)

$$
\overline{u_{i}^{\prime} T^{\prime}}=-\alpha_{t} \frac{\partial T}{\partial x_{i}}=-\frac{\mu_{t}}{\operatorname{Pr}_{t}} \frac{\partial T}{\partial x_{i}}
$$

where $\alpha_{t}$ and $\operatorname{Pr}_{t}$ are the thermal diffusivity and turbulent Prandtl number respectively. A non-linear extension of this is the generalised gradient diffusion hypothesis (GGDH) [20]

$$
\overline{u_{j}^{\prime} T^{\prime}}=-\frac{f}{\omega} \tau_{i j} \frac{\partial T}{\partial x_{j}}
$$

where $f=f\left(I_{m}, J_{n}\right)$ is a scalar field to be optimised, with $I_{m}$ and $J_{n}$ described by Eqns. (3) and (7) respectively. While the GGDH does perform better in representing the heat-flux, for the sake of simplicity, we start by using the SGDH in this study and build a model instead for $1 / \mathrm{Pr}_{t}$ in Eqn. (4). Following the same approach used to the split the Reynolds stress tensor [14], the heatflux vector can be split into the corresponding deterministic and stochastic contributions using triple decomposition [21] as

$$
\begin{aligned}
\overline{u_{j}^{\prime} T^{\prime}} & =\overline{\left(\widetilde{u}_{j}+u_{j}^{\prime \prime}\right)\left(\widetilde{T}+T^{\prime \prime}\right)}, \\
& =\overline{\widetilde{u}_{j} \widetilde{T}+u_{j}^{\prime \prime} \widetilde{T}+\widetilde{u}_{j} T^{\prime \prime}+u_{j}^{\prime \prime} T^{\prime \prime}}, \\
& =\overline{\widetilde{u}_{j} \widetilde{T}}+\overline{u_{j}^{\prime \prime} T^{\prime \prime}} .
\end{aligned}
$$

Here, a variable $\Phi=\bar{\Phi}+\widetilde{\Phi}+\Phi^{\prime \prime}$ such that $\bar{\Phi}, \widetilde{\Phi}$ and $\Phi^{\prime \prime}$ represent the mean, the deterministic and the stochastic components of $\Phi$ respectively. Also, $\widetilde{\Phi}+\Phi^{\prime \prime}=\Phi^{\prime}$ where $\Phi^{\prime}$ is the Reynoldsaveraged fluctuating component of $\Phi$.

With this split performed, the SGDH closure for the stochastic heat-flux can be developed for $1 / \operatorname{Pr}_{t}$ using the scalar invariants, i.e. $1 / \operatorname{Pr}_{t}=f\left(I_{m}, J_{n}\right), \forall m \in[1,2]$ and $\forall n \in[1,5]$ where

$$
\begin{aligned}
J_{1} & =\frac{\partial T}{\partial x_{i}} \frac{\partial T}{\partial x_{i}}, \quad J_{2}=\frac{\partial T}{\partial x_{i}} S_{i j} \frac{\partial T}{\partial x_{j}}, \quad J_{3}=\frac{\partial T}{\partial x_{i}} S_{i j} S_{j k} \frac{\partial T}{\partial x_{k}} \\
J_{4} & =\frac{\partial T}{\partial x_{i}} \Omega_{i j} \Omega_{j k} \frac{\partial T}{\partial x_{k}}, \quad J_{5}=\frac{\partial T}{\partial x_{i}} \Omega_{i j} S_{j k} \frac{\partial T}{\partial x_{k}}
\end{aligned}
$$

The summary of the machine-learning approach is shown in the schematic in Fig. 1, which has been modified to include the heat-flux vector processing.

\section{NUMERICAL SETUP}

In this section, we describe the setup of both the solver used to generate the high-fidelity database as well as the solver chosen to conduct the URANS with the new closures. The dataset for the machine-learning or "training" process was obtained by conducting three high-resolution LES using the WALE model [22] with the in-house solver HiPSTAR [23]. The solver is a finitedifference based code solving the non-dimensional compressible Navier-Stokes equations for density, momentum and total energy using a fourth-order accurate stencil for spatial discretisation and a fourth-order accurate low storage Runge-Kutta scheme 
TABLE 1: Simulation Parameters

\begin{tabular}{c|c}
\hline \hline Parameters & Values \\
\hline \hline$U_{\text {bulk }}$ & $16.5,20.5,24.2 \mathrm{~m} / \mathrm{s}$ \\
\hline$U_{f s}$ & $19.2 \mathrm{~m} / \mathrm{s}$ \\
\hline$l_{t}$ & $10 \%$ of slot height \\
\hline $\mathrm{BR}$ & $0.86,1.07,1.26$ \\
\hline$t / s$ & 1.14 \\
\hline$R e_{\text {slot }}$ & 12,000 \\
\hline$T_{\text {slot }}$ & $273 \mathrm{~K}$ \\
\hline$T_{f s}$ & $323 \mathrm{~K}$ \\
\hline \hline
\end{tabular}

$90 s$

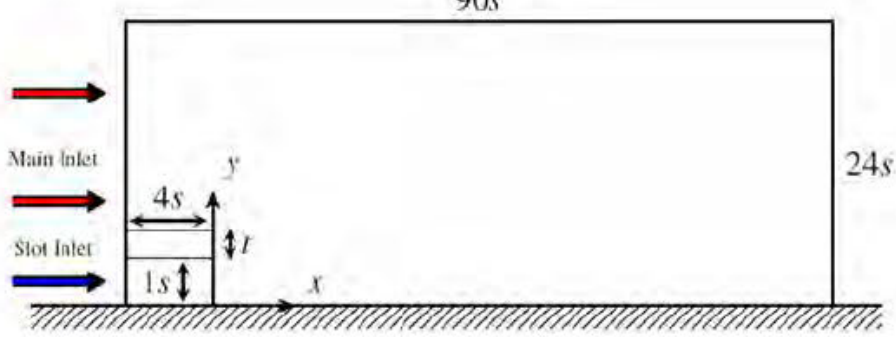

FIGURE 2: Schematic of the trailing-edge slot configuration

for temporal discretisation. The simulations were performed with a reference Mach number of 0.25 , Prandtl number of 0.75 and Reynolds number of 9,134, based on the slot thickness and freestream velocity. The LESs were subsequently benchmarked with the experimental data from Kacker \& Whitelaw [1]. It was also observed that the subgrid scale stresses were negligible compared with the resolved stresses, thus only the resolved components were used for the "training" process. The details of the domain and grid resolution are provided in Sandberg et al. [10], with the domain schematic shown in Fig. 2. The schematic shows a co-flow separated by a lip of finite thickness $t=1.14 \mathrm{~s}$, where $s=1$ is the slot height. $t=1.14 \mathrm{~s}$ corresponds to the thick lip case and has been chosen as it exhibits strong vortex shedding. An improvement in this case was harder to realise in Sandberg et al. [10] on account of RANS not capturing the vortex shedding, making it a suitable case to test the unsteady framework of this study. The slot ejects fluid at a temperature of $273 \mathrm{~K}$ forming a film along the lower wall with the velocity of ejection defined in relation to the freestream velocity in the main flow, which is at a temperature of $323 \mathrm{~K}$. The LESs were performed without any inflow turbulence fluctuations since Ivanova \& Laskowski [4] showed that the results downstream of the slot exit were very similar with/without fluctuations at the inlet, the transition being dominated by the strong instability in the mixing layer instead of the turbulent fluctuations. Three blowing ratios (BRs) are considered in this study with values of $0.86,1.07$ and 1.26. In this study, BR is defined as the ratio of the bulk velocity $\left(U_{b}\right)$ of the slot flow to the freestream velocity $\left(U_{f s}\right)$ in the main flow instead of the streamwise flux ratios, consistent with previous studies $[10,14]$. The bulk velocity is obtained by integrating the mean velocity profile w.r.t the slot height. Given the BRs, the flow can be classed as a wall-jet for BR $>1.0$ and wall-wake for $B R<1.0$ [24]. The length, temperature and velocity scales are non-dimensionalised with $s, T_{f s}$ and $U_{f s}$ respectively. The simulation parameters have been summarised in Table1.

The URANS calculations were performed with the opensource code OpenFOAM, which is a finite-volume Navier-Stokes solver. The compressible solver rhoP impleFoam was utilised, solving for the mass, momentum and enthalpy equations while the turbulence model used was the compressible $k-\omega$ SST [16]. The calculations were conducted with a timestep of 0.005 , leading to a maximum Courant number of just under 1 while the PIMPLE loop utilised one outer loop and two inner correctors for the pressure equation. To remain consistent with the LES, the Sutherland transport was used to calculate the molecular viscosity, however, the sutherland transport dictionary also sets up the thermal diffusivity $(\alpha)$, which is constructed using the modified Eucken formula [25], i.e. $\alpha=\mu(1.32+1.77(\gamma-1) / \gamma)$, which leads to a molecular Prandtl number of 0.693 for $\gamma=1.4$. Thus, an offset is added to $\alpha$ such that the molecular Prandtl number is consistent with the LES of 0.75 . Furthermore, to eliminate any upstream errors, the velocity, $k$ and $\omega$ profiles from the LES were fed to the solver as inflow boundary conditions. The machine-learnt models were implemented in the $k-\omega$ SST solver by building the Reynolds stress tensor and the turbulent thermal diffusivity using

$$
\begin{aligned}
\tau_{i j} & =\frac{2}{3} k \delta_{i j}-2 \frac{\mu_{t}}{\rho} S_{i j}+2 k a_{i j}^{x}, \\
\alpha_{t} & =\mu_{t} f\left(I_{m}, J_{n}\right),
\end{aligned}
$$

where $a_{i j}^{x}=a_{i j}^{m o d}+V_{i j}^{1}$ is the extra anisotropy (split this way to ensure solver stability) and $f\left(I_{m}, J_{n}\right)$ is the model for $1 / \mathrm{Pr}_{t}$. In this formulation $\mu_{t}=\rho a_{1} k / \max \left(a_{1} \omega, S F 2\right)$, where $a_{1}=0.31$ and $S F 2=\sqrt{2 S_{i j} S_{i j}} \tanh \left[\left(\max \left(2 \sqrt{k} / \beta^{*} \omega y\right), 500 v / y^{2} \omega\right)^{2}\right]$, with $\beta^{*}=0.09$ and $y$ the distance from the wall.

Before proceeding with the simulations, a grid convergence test was performed to obtain the optimal grid without compromising the results. The domain extent for the URANS was kept the same as the LES with successive grids tested being coarser than the LES resolution in the plane $\left(x_{1}-x_{2}\right)$ and the spanwise $\left(x_{3}\right)$ directions. The criteria for considering a grid optimal was a comparison of the mean streamwise profiles of the velocity across the successive grids, until the results deviated from the URANS solution on the LES grid. Thus, the optimal grid was coarser than the LES grid by a factor of 2 in both the $x_{1}$ and the $x_{2}$ directions and by a factor of 4 in the $x_{3}$ direction, resulting in $800 \times 384 \times 16$ points. The resulting computational expense for obtaining a time-converged solution equivalent to 3 flow-through times was found to be 864 core-hours (144 cores for 6 hours) for the URANS as opposed to 23,410 core-hours (1608 cores for 14.56 hours) for the LES. This means the URANS presented in the following sections is 27 times cheaper than the LES, even though the LES was performed with an optimised high-fidelity code. This factor could be significantly larger had the same LES been run in OpenFOAM. 


\section{RESULTS}

This section is split into three parts. First, we implement the closure for the anisotropy in the URANS solver and conduct calculations of the $B R=1.26$ case. Second, we build a closure for $1 / \mathrm{Pr}_{t}$ on the $\mathrm{BR}=1.26$ case and implement the model into the URANS solver. Finally, we apply the closures for the anisotropy and heat-flux to the BR $=0.86$ and 1.07 cases to test the generalisability of the closures. While the Reynolds stress and turbulent heat-flux are correlated, we perform the training of each quantity individually, since previous research efforts $[10,26]$ demonstrate heat-flux closure development independently of the stress-strain closure development which allows the decoupling of the momentum equation from the energy equation.

\section{Turbulence stress closure perfomance}

It was shown in Lav et al. [14] that the flow in the trailingedge slot configuration was essentially a wall-jet/wake depending on the BR and thus, required building of two closures for a given $\mathrm{BR}$, one for the near wall boundary layer and one away from the wall for the jet/wake flow, i.e. a two-zone model. It was also shown a priori that the closure developed for $\mathrm{BR}=1.26$ performed well on itself and the other BRs, i.e. 0.86 and 1.07. This closure is given by

$$
\begin{aligned}
a_{i j}^{\text {mod, } B} & =\left(I_{1}-I_{2}\left(I_{1}-3 I_{2}\right)-0.53\right) V_{i j}^{1} \\
& -\left(2 I_{2}-4.85\right)\left(2 I_{2}-1\right) V_{i j}^{2} \\
& +\left(-5 I_{1}+5 I_{2}+4.518\right) V_{i j}^{3}, \\
a_{i j}^{\text {mod }, J} & =\left(I_{1}-I_{2}-0.71\right) V_{i j}^{1} \\
& +\left(I_{1}\left(I_{1}+I_{2}\right)\left(2 I_{1}-I_{2}\right)-0.71 I_{1}+0.5041\right) V_{i j}^{2} \\
& +\left(I_{1}\left(I_{2}-2\right)\left(I_{1}-I_{2}+0.73\right)-4 I_{1}+2 I_{2}+4.71\right) V_{i j}^{3},
\end{aligned}
$$

where superscripts $B, J$ refer to the boundary layer and jet models. Implementing these closures in URANS means incorporating a switching function such that only the boundary layer closure works near the wall and only the jet closure works away from the wall. It was found that the demarcation curve between the two closures corresponded to the location where $\partial \overline{u_{1}} / \partial x_{2}$ was maximum and that these locations were similar across the different BRs. Thus, a switching function was built, as shown in Fig. 3 where the blue region corresponds to the $a_{i j}^{\bmod , B}$ closure while the red region corresponds to the $a_{i j}^{\text {mod }, J}$ closure. The switch employs a ramping function such that the transition between the closures occurs smoothly over 5-10 grid points.

The switching function is constructed using the pos () function which returns 1 for non-negative arguments, where the argument is set as the demarcation curve, i.e. the cubic polynomial expression. Thus, generalising the switch for any functional

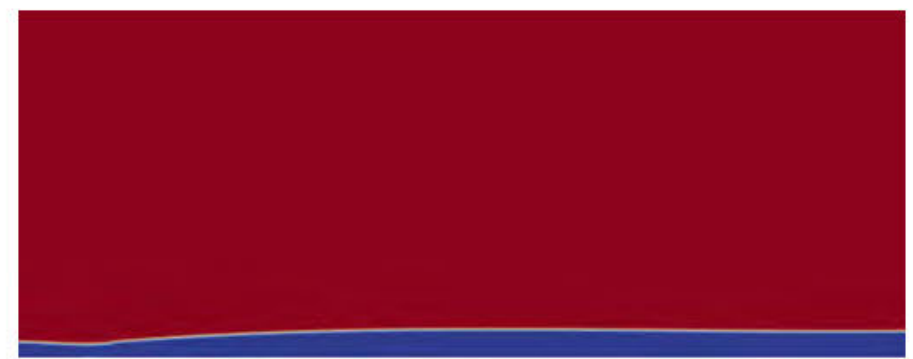

(a)

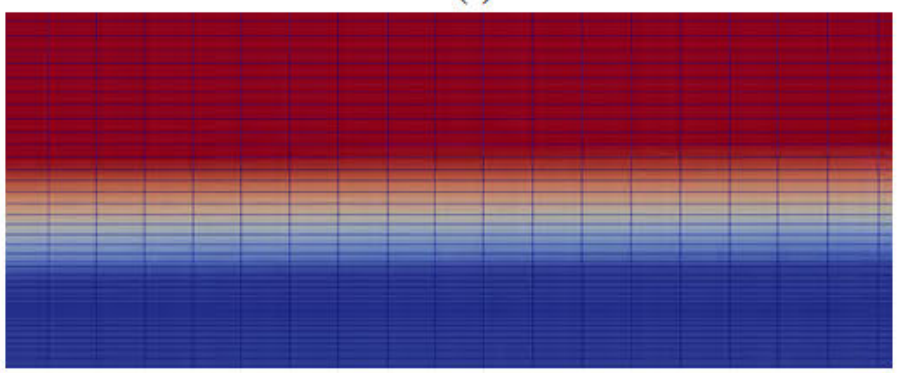

(b)

FIGURE 3: (a) Switching function showing two regions immediately downstream of the slot exit (b) Closeup of region where switching occurs, along with the grid resolution.

$$
\text { form } \begin{aligned}
x_{2}= & f\left(x_{1}\right): \\
\Psi_{1} & =\operatorname{pos}\left(x_{2}-f\left(x_{1}\right)-0.1\right), \\
\Psi_{2} & =\frac{\operatorname{pos}\left(x_{2}-f\left(x_{1}\right)+0.1\right)-\operatorname{pos}\left(x_{2}-f\left(x_{1}\right)-0.1\right)}{0.2}, \\
\text { swt ch } & =\Psi_{1}+\left(x_{2}-f\left(x_{1}\right)+0.1\right) \Psi_{2}, \\
a_{i j}^{\text {mod }} & =(1-\operatorname{swtch}) a_{i j}^{\text {mod, }, B}+(\operatorname{swtch}) a_{i j}^{\text {mod, },} .
\end{aligned}
$$

Here, the term $\Psi_{1}$ refers to the demarcation curve, shifted by -0.1 units while the term $\Psi_{2}$ refers to the ramped part of the switch which smoothly varies from 0 to 1 over a distance of 0.1 to 0.1 units across $x_{2}=f\left(x_{1}\right)$ in the $x_{2}$ direction. The total switching function is then given by swtch where the offset of 0.1 units was chosen because it allowed upto 10 grid points across the ramp region, as evident from Fig. 3. For a start, the two-zone model was applied throughout the domain and calculations were conducted for the $\mathrm{BR}=1.26$ case. A baseline URANS with the Boussinesq approximation (called untrained) was also conducted for comparison, however, it was observed that the baseline URANS suppressed the vortex shedding, leading to a steady solution. A number of approaches were experimented with, such as grid refinement to levels finer than the LES resolution, higher order spatial discretisation schemes (from the second-order linearUpwind to the fourth-order cubic) and fluctuating inflow conditions, similar to the suggestion of Holloway et al. [7], where, using the turbulent Inlet boundary condition, the mean inflow profiles of velocity and pressure were overlayed with a random component of $1 \%$ intensity. Between these choices, the latter two were successful in sustaining the shedding. However, the unfiltered higher-order scheme introduced point-point oscillations while the fluctuating inflow introduced non-physical smaller structures and so, neither ap- 

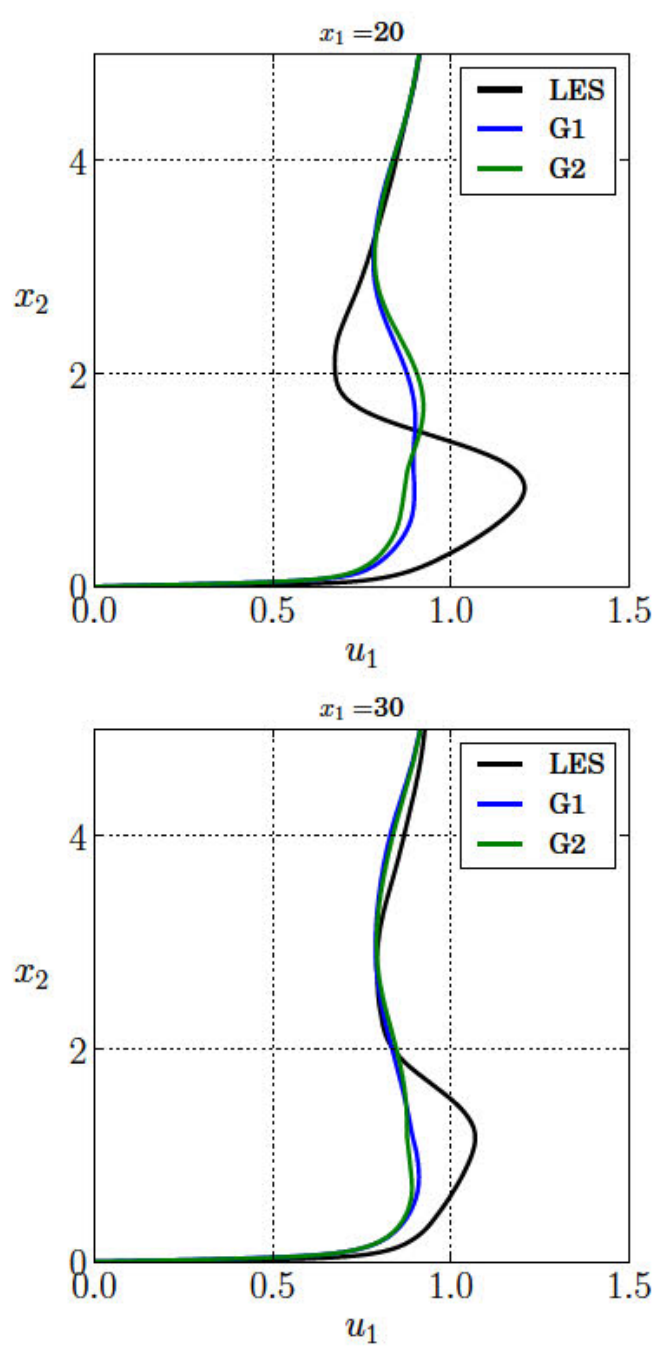

FIGURE 4: Grid independence test of a trained URANS calculation showing the streamwise velocity profile between the optimal grid G1 $(800 \times 192 \times 16)$ and LES grid G2 $(1600 \times 384 \times 66)$.

proaches were considered. Both the trained and untrained calculations were performed with the SGDH closure using $\operatorname{Pr}_{t}=0.9$. Since the calculations with the machine-learnt closures incorporate gradients of the temperature and velocity, a grid independence test was again conducted between the optimal URANS grid (labelled G1) and the LES grid (labelled G2) resolutions. The result of this test is presented in Fig. 4, which shows that the optimal URANS grid has insignificant deviations from the results on a much finer grid resolution. Figure 5 shows the mean streamwise velocity contours for comparison at the near slot exit between the LES and trained URANS. The figure shows that the trained calculation leads to a "lift-off" in the flow downstream of the slot exit, which leads to a poor prediction of the wall temperature. This means the two-zone closure when applied everywhere introduces an error in the near slot exit region, which affects the flow conditions further downstream as well. Since the region used to develop this closure spanned 5-30 units in $x_{1}$ [14], a second calculation with the trained closure was performed where all modelling for the Reynolds stress was switched-off up to $x_{1}=$ 5 units and the two-zone model switched-on after $x_{1}=5$ units.

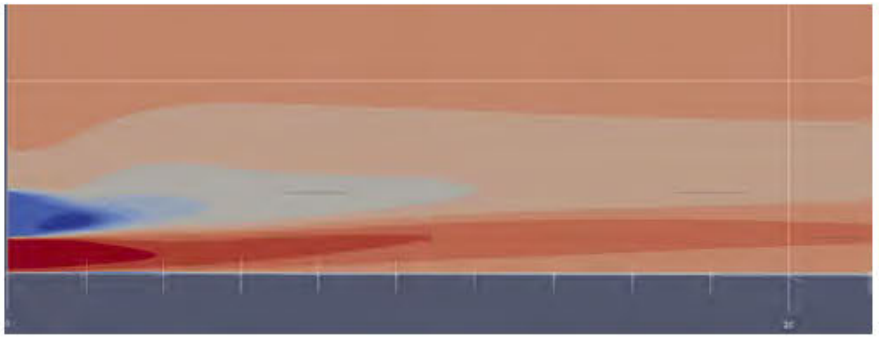

(a)

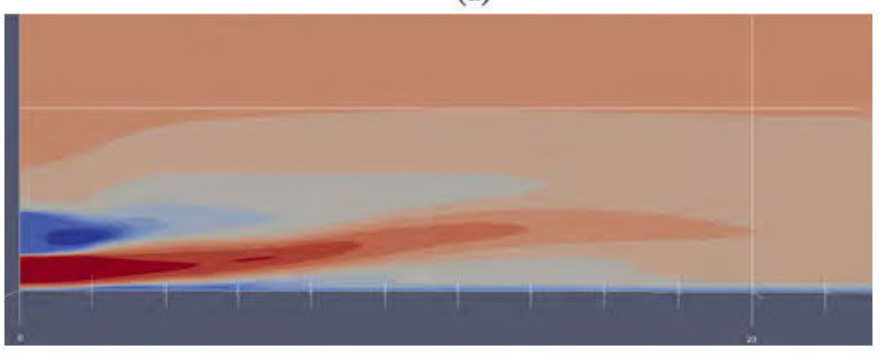

(b)

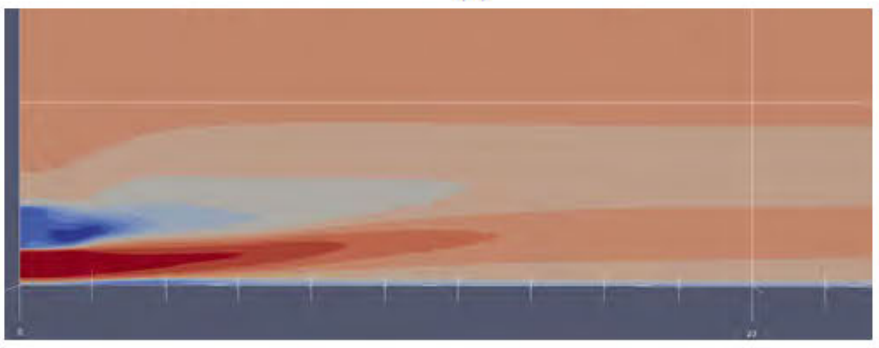

(c)

FIGURE 5: Mean streamwise velocity contours for (a) LES, (b) trained URANS running two-zone closure everywhere, (c) trained URANS running two-zone model applied after $x_{1}=5$ and with no modelling upstream.

The streamwise velocity contours for this case are also shown in Fig. 5 and there is a marked improvement in the contours when compared to the reference LES. Figure 6 shows the streamwise velocity profiles for the untrained and trained URANS with the reference LES at three different locations, highlighting the improvement in the profile shape with the switching-off of the modelling prior to $x_{1}=5$ units. The decision to switch-off all modelling prior to $x_{1}=5$ is grounded in the physics of this flow configuration. In order to understand this, we can construct a quantity $f_{k}^{*}=k^{\prime \prime} / k$ from the LES dataset, which refers to the ratio of the stochastic turbulence kinetic energy to the total turbulence kinetic energy, i.e. the proportion of stochastic scales to the total scales in the flow. The terms $k$ and $k^{\prime \prime}$ are both obtained from the resolved LES datasets, with $k^{\prime \prime}$ obtained by subtracting the deterministic contribution, obtained via the triple decomposition, from the resolved $k$ field. Thus, $f_{k}^{*}=1$ indicates the presence of only stochastic scales while $f_{k}^{*}=0$ indicates the presence of only deterministic scales. Figure 7 shows the contours for $f_{k}^{*}$ for all three BRs and it is clearly visibile that $f_{k}^{*}$ is very small at the exit of the slot, particularly up to $x_{1}=5$. Only then, $f_{k}^{*}$ starts to increase and reaches 1 downstream of $x_{1}=30$. These contours demonstrate that near the slot exit, the length scales are purely deterministic, coming from the vortex shedding. As the flow then develops downstream, the shedding scales break down 

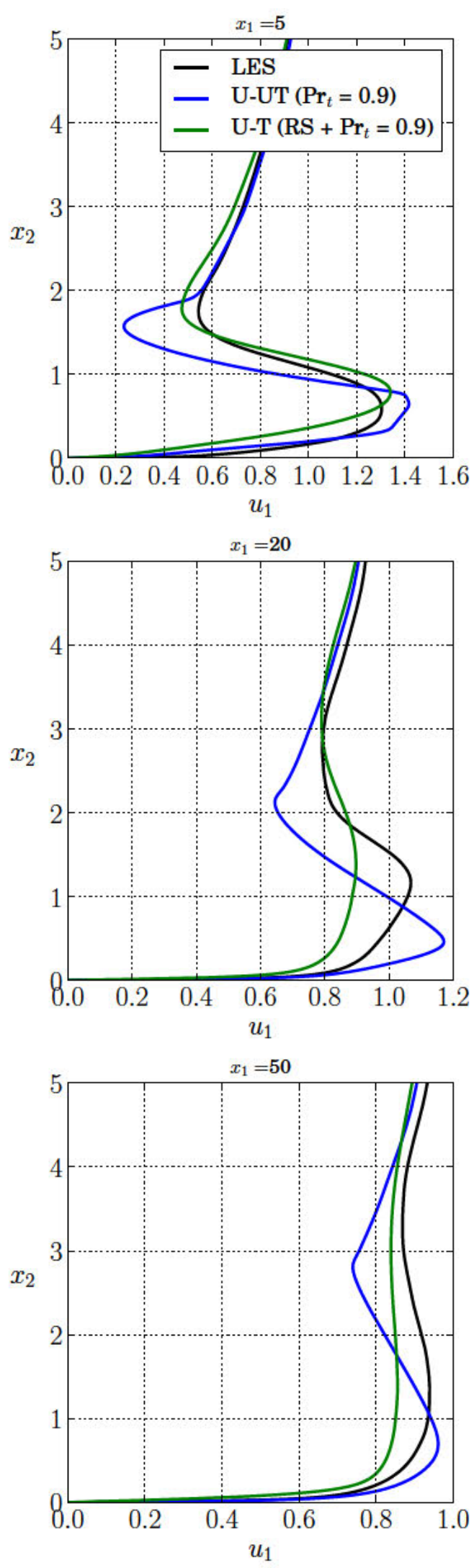

FIGURE 6: Streamwise velocity profiles at three different locations comparing baseline URANS and trained URANS with the reference LES.

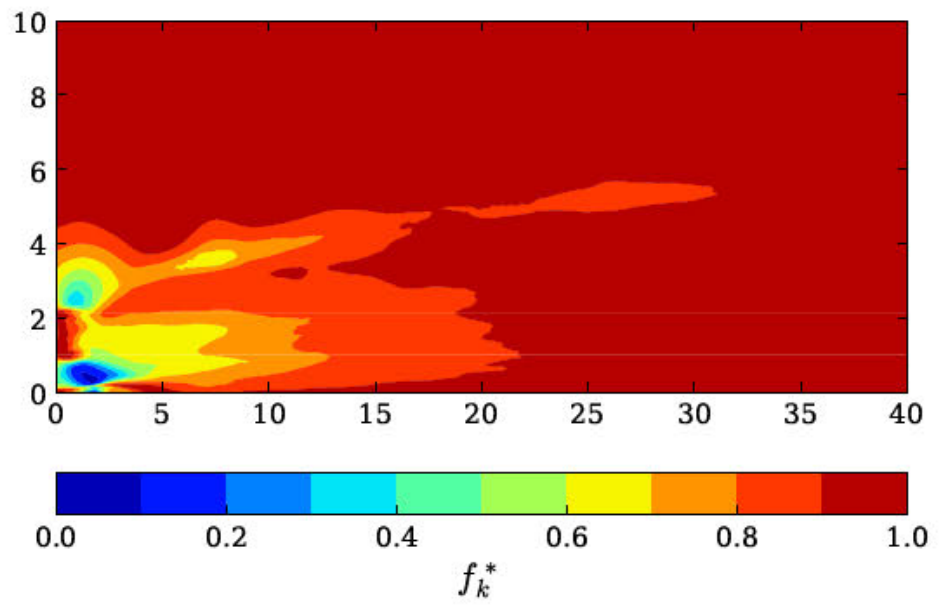

(a)

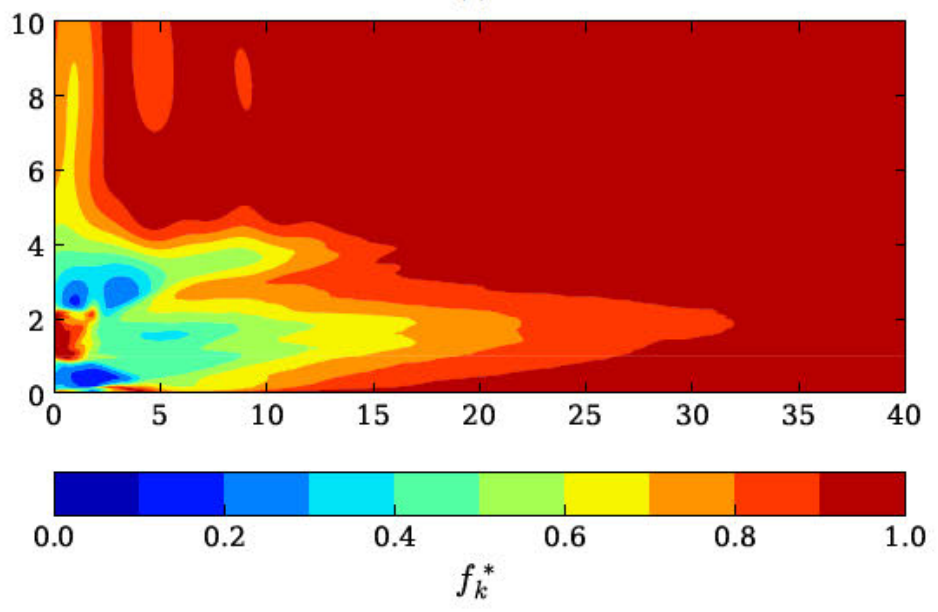

(b)

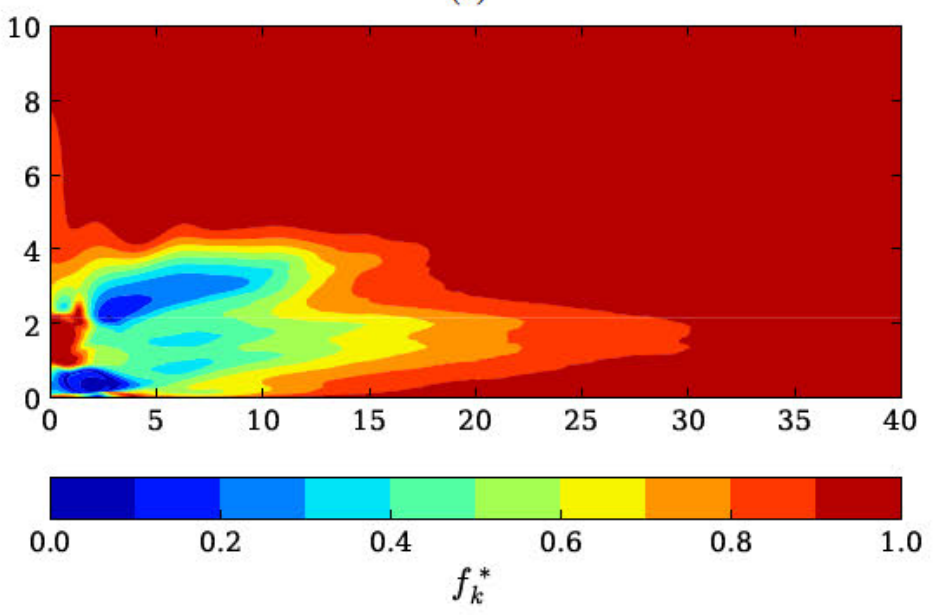

(c)

FIGURE 7: Spatial distribution of $f_{k}^{*}=k^{\prime \prime} / k$ from the LES datasets for (a) $\mathrm{BR}=0.86$, (b) $\mathrm{BR}=1.07$, (c) $\mathrm{BR}=1.26$.

leading to increased presence of stochastic scales, finally leading to purely stochastic scales after $x_{1}=30$. Since we are resolving the vortex shedding in the near slot exit with the calculations and these are the only scales present (note that this is because the current simulation setup does not consider inflow turbulence fluctuations), adding a closure for the stochastic scales through 


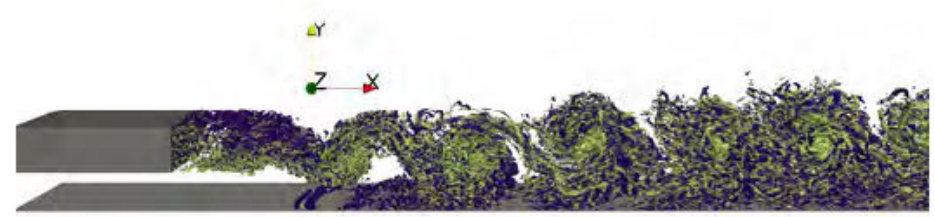

(a)

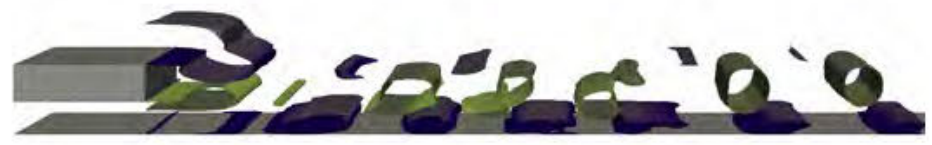

(b)

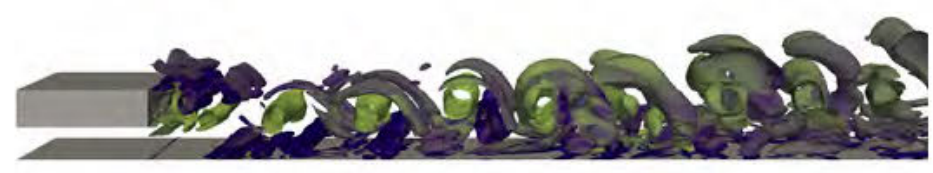

(c)

FIGURE 8: Instantaneous Q-criterion of the BR $=1.26$ case for (a) LES (b) untrained URANS and (c) trained URANS.

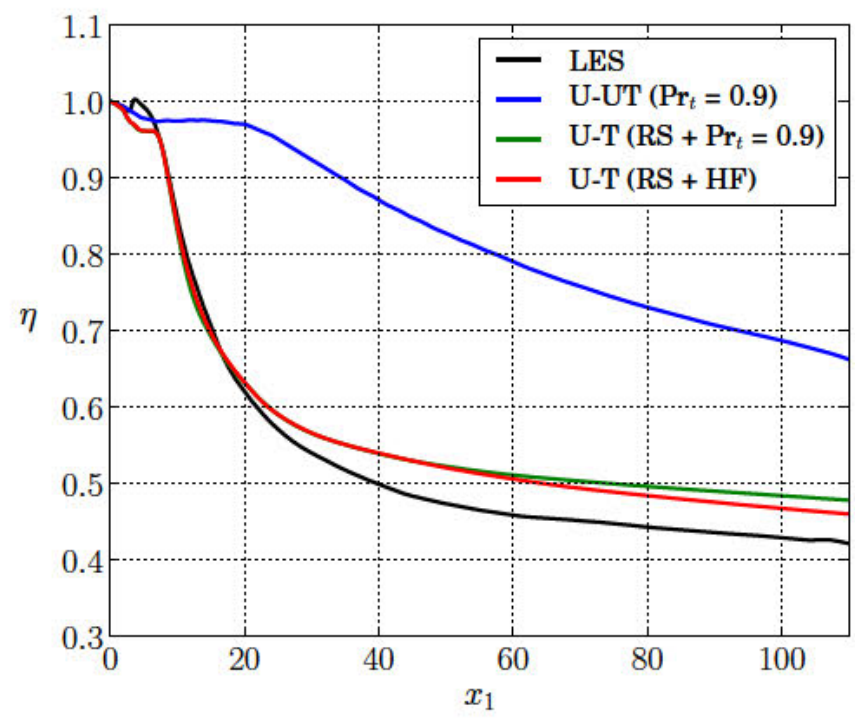

FIGURE 9: Adiabatic wall-effectiveness for $B R=1.26$ case. $\mathrm{U}$ : URANS, UT: Untrained, T: Trained.

the turbulence stress is unnecessary and downright detrimental to predicting the flow. The result of running this configuration of models is demonstrated through an instantaneous snapshot of the Q-criterion [27] in Fig. 8, which shows the flow structures of the reference LES, the untrained URANS and the trained URANS. Clearly, the trained model does a far superior job at resolving the vortex shedding, in addition to resolving extra length scales in and around the rollers as well as structures near the wall. This improvement also translates to a significantly better prediction of the adiabatic wall-effectiveness, as shown in Fig. 9, where the legend key U-T (RS) represents the trained model run with the zonal closure for the turbulence stress with a $\operatorname{Pr}_{t}=0.9$. In the

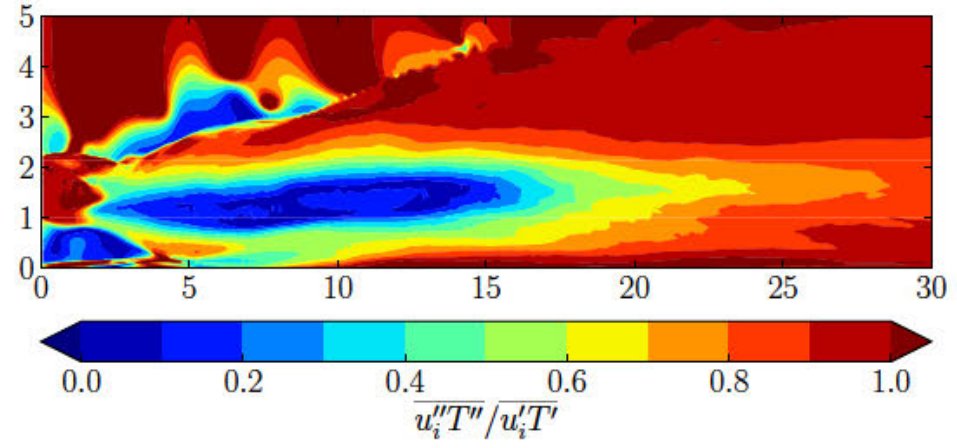

FIGURE 10: Ratio of stochastic to total heat-flux obtained from the LES dataset for $\mathrm{BR}=1.26$ case. Both contributions in the contour are summed over all three flux vector components.

immediate vicinity of the slot exit, we observe both trained and untrained calculations underpredict $\eta$, which is possibly due to lack of resolution of a small closed separation bubble present in the LES.

\section{Heat-flux closure evaluation}

The improvement in $\eta$ predicted by the new turbulence stress closure is remarkable, particularly in the near slot exit region with deviations from the reference data occurring only after $x_{1}=25$ units. To realise improvements in this downstream region, we now turn our attention to developing a new heat-flux closure that still uses the SGDH. As shown in Eqn. (6), we first split the heat-flux vector to obtain the stochastic contribution. Figure 10 shows the spatial contours of the ratio of stochastic to total heat-flux following the split, to demonstrate the strong contribution of the deterministic scales even in the heat-flux. The spatial distribution, however, is different from the contours in Fig. 7, which is a good indicator of the weak correlation between the turbulent stress and the turbulent heat-flux, justifying the decision to train closures for each quantity individually. As was done in the turbulence stress closure development, we choose the same spatial region for training, i.e. $x_{1} \in[5,30]$ units and $x_{2} \in[0,5]$ units and perform scalar regression using GEP to obtain a model $f\left(I_{m}, J_{n}\right)$ such that

$$
\overline{u_{i}^{\prime \prime} T^{\prime \prime}}+f\left(I_{m}, J_{n}\right) \mu_{t} \frac{\partial T}{\partial x_{i}}=0
$$

where $\overline{u_{i}^{\prime \prime} T^{\prime \prime}}$ and $\mu_{t} \partial T / \partial x_{i}$ are taken from the LES dataset. The resulting function obtained after running the algorithm 50 times with different initialisations yields

$$
\begin{aligned}
f\left(I_{m}, J_{n}\right) & =I_{1}^{2} I_{2}-4 I_{1} I_{2}+15 I_{1} J_{1}-I_{1} J_{2}+0.939 I_{1} \\
& +8 I_{2}+J_{1}^{2}-88.736 J_{1}+4 J_{2}+2.12349 .
\end{aligned}
$$

As can be seen, this function is composed of the invariants described in Eqns. (3) and (7) in addition to a constant. Since $f\left(I_{m}, J_{n}\right)$ approximates $1 / \operatorname{Pr}_{t}$, this shows that the machine-learnt closure is suggesting a "mean" $\operatorname{Pr}_{t}$ of 0.471 , which is lower than 
the usual value of 0.9 . The value of $1 / \operatorname{Pr}_{t}$ varies around 0.471 depending on the sign and magnitude of the invariants $I_{m}, J_{n}$. Calculations were then run on the $\mathrm{BR}=1.26$ case by coupling the turbulence stress and heat-flux closures and the resulting $\eta$ is shown in Fig. 9 with the legend U-T (RS + HF). Key takeaways from this plot that can be summarised as

1. The addition of the heat-flux closure produced results similar to the $\operatorname{Pr}_{t}=0.9$ case up to $x_{1}=50$ and shows an improvement in $\eta$ after this position. The region beyond $x_{1}=50$ is purely dominated by stochastic scales, so a tailored stochastic closure leads to the additional improvement there.

2. The similar behaviour upstream of $x_{1}=50$ shows that the improvement in $\eta$ is dominated by the closure for the turbulence stress, instead of the heat-flux. This is directly related to the improved resolution of the vortex shedding and the existence of these shedding scales leads to enhanced mixing and the steep drop in $\eta$.

Even though the improvement achieved by the heat-flux closure is marginal over the improvement due to the turbulence stress closure, it is important to keep in mind that these calculations are 27 times cheaper than an LES due to a significantly coarser grid, yet manage to close the gap to the LES, such as bringing down the error in $\eta$ from $75 \%$ in case of the baseline URANS to $10 \%$ with the trained calculation at $x_{1}=40$.

\section{Generalisability of closures}

With the improvements observed in the previous section, in this section, the closures for the turbulence stress and the heatflux developed for the BR $=1.26$ are applied "as is" on different BRs, i.e. 0.86 and 1.07. While the latter ratio corresponds to a wall-jet, similar to the $B R=1.26$ case, the former is classed as a wall-wake which produces fundamentally different flow physics away from the wall [2]. Figure 11 shows $\eta$ for the two additional BRs, where similar to the $\mathrm{BR}=1.26$ case, three calculations were performed. The untrained baseline again shows a significant level of overprediction whereas the machine-learnt models perform significantly better than the baseline models, managing to capture the right spatial location where the sharp drop in $\eta$ occurs. It is reassuring that the closure for the turbulence stress consistently improves the results across the different BRs. In case of the calculation also using the machine-learnt heat-flux, the results are not as encouraging. The addition of the heat-flux closure results in an overprediction of $\eta$ over the calculation using the stress closure with $\operatorname{Pr}_{t}=0.9$, which is rather significant for the $\mathrm{BR}=0.86$ case. These results indicate that $\mathrm{Pr}_{t}$ obtained for the $\mathrm{BR}=1.26$ case is not optimal for the $\mathrm{BR}=0.86$ and 1.07 cases. Thus, we also develop closures for the heat-flux based on the LES data for $\mathrm{BR}=0.86$ and 1.07 , with the resulting expressions being

$$
\begin{aligned}
& \mathbf{B R}=\mathbf{0 . 8 6 :} \\
& \quad \begin{aligned}
f\left(I_{m}, J_{n}\right) & =-10.94 I_{1}+9 I_{2}-11.97 J_{1} \\
& +4\left(2 J_{1}+J_{2}\right)\left(I_{1}+4 J_{1}-1.94\right)+2.517, \\
\mathbf{B R}=1.07: & \\
f\left(I_{m}, J_{n}\right) & =-I_{1}^{2}+18 I_{1} I_{2}-8 I_{1}-13 I_{2}^{2}-I_{2} J_{3}+I_{2} J_{4} \\
& +18.52 I_{2}+3 J_{1}-3 J_{2}-2 J_{4}-3 J_{5}+1.75 .
\end{aligned}
\end{aligned}
$$

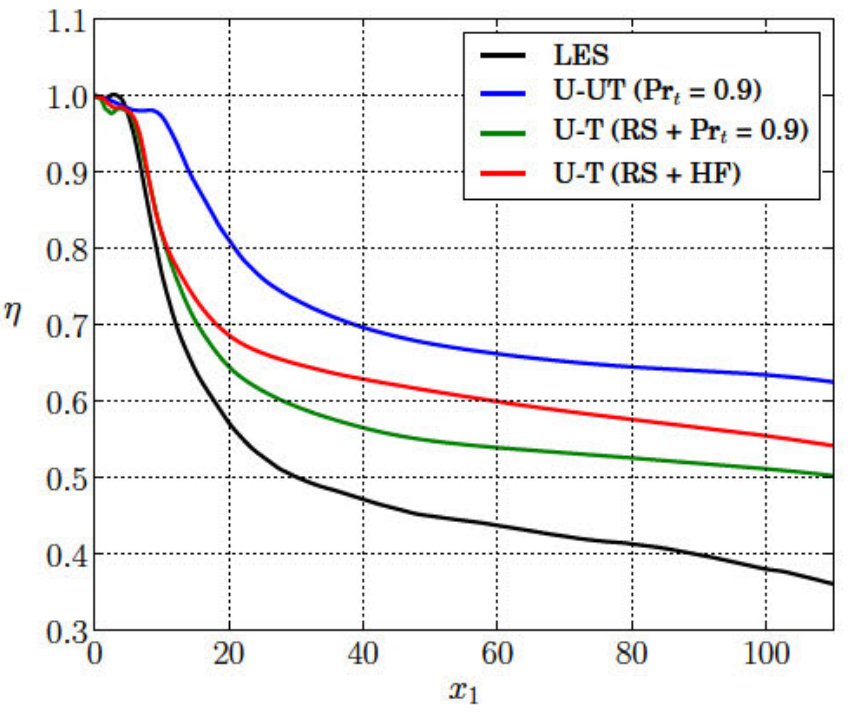

(a)

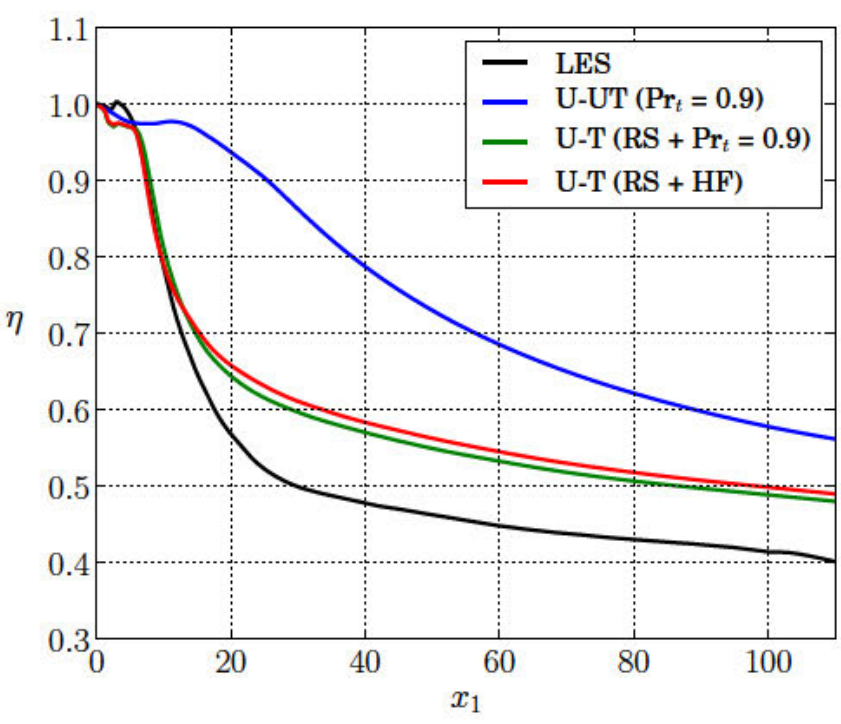

(b)

FIGURE 11: Generalisability test on the adiabatic walleffectiveness for (a) $\mathrm{BR}=0.86$ and (b) $\mathrm{BR}=1.07$ cases.

These closures indicate that the "mean" $\mathrm{Pr}_{t}$ would be 0.397 for $\mathrm{BR}=0.86$ and 0.571 for $\mathrm{BR}=1.07$, which are both different from the $B R=1.26$ case. However, calculations with these new closures were not conducted for two reasons. First, the aim is to build a generalisable closure that can work on all BRs and having a heat-flux closure for every BR is not a sustainable approach. Second, even running the calculation with the optimal heat-flux closure will not significantly outperform the trained calculation with $\operatorname{Pr}_{t}=0.9$, as was seen from Fig. 9 for the $\mathrm{BR}=1.26$ case. While the results are encouraging with the marked improvement in $\eta$, the calculations also highlight that the use of the standard gradient diffusion hypothesis (Eqn. 4) is evidently not appropriate as it does not produce desired improvements across different operating conditions. Alternatively, the generalised gradient diffusion hypothesis (Eqn. 5) could be a suitable choice. The 

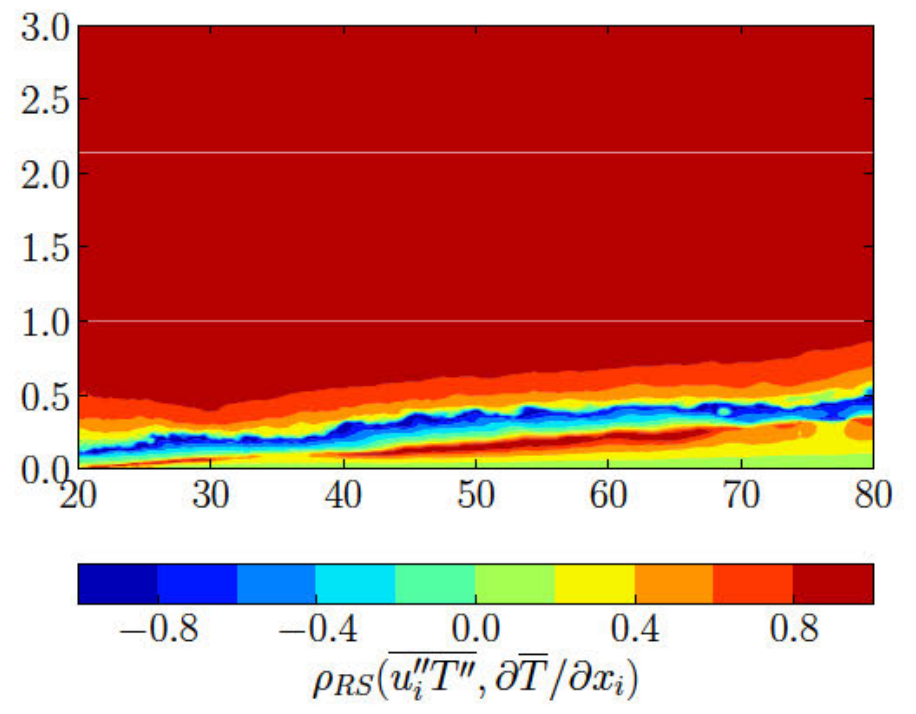

(a)
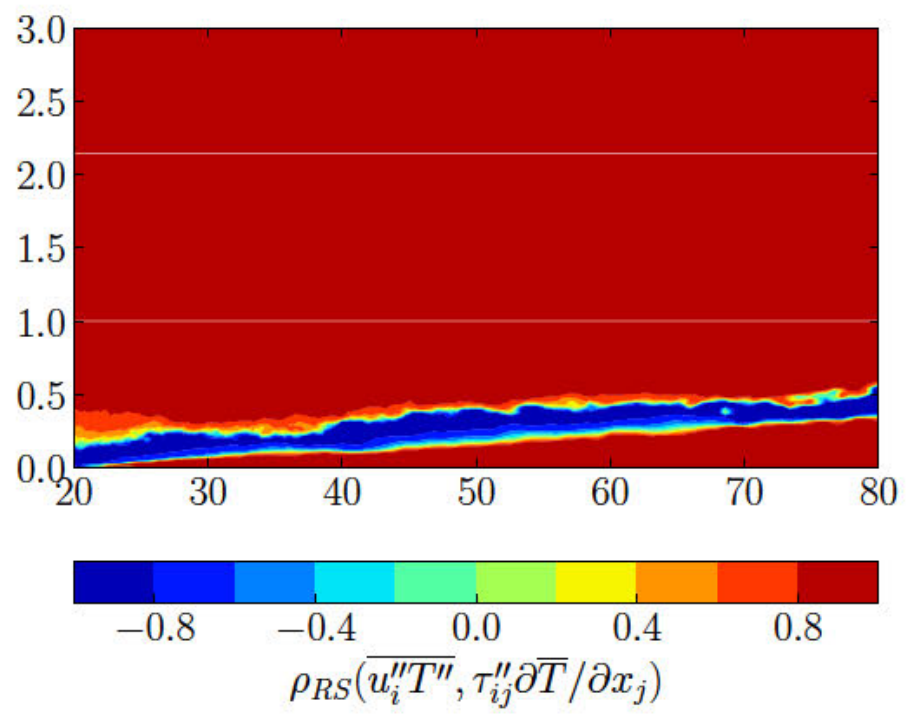

(b)

FIGURE 12: Alignment of the stochastic heat-flux vector with the modelled heat-flux vector using (a) SGDH and (b) GGDH. All quantities taken from reference LES at $B R=1.26$. The contours span $[20,80]$ units in the $x_{1}$ and $[0,3]$ units in the $x_{2}$ directions.

difference between the two hypotheses can be illustrated in an a priori sense by computing the alignment between the heatflux vector and the hypothesis, similar to the alignment plots for the anisotropy closures [14], shown in Fig. 12. The alignment $\rho_{R S}\left(L_{i}, M_{i}\right)$ can be defined as

$$
\rho_{R S}\left(L_{i}, M_{i}\right)=\frac{L_{i} M_{i}}{\sqrt{L_{p} L_{p} M_{q} M_{q}}},
$$

where $\rho_{R S}\left(L_{i}, M_{i}\right) \in[-1,1]$ and indicates the angle between the vector $L_{i}$ and $M_{i}, \forall i \in[1,3]$. In this instance, $L_{i}=u_{i}^{\prime \prime} T^{\prime \prime}$, while $M_{i}=\partial \bar{T} / \partial x_{i}$ for the GDH or $M_{i}=\tau_{i j}^{\prime \prime} \partial \bar{T} / \partial x_{j}$. If the alignments are $1,-1$ and 0 , the vectors are perfectly, oppositely and orthogonally aligned with one another, respectively. While the ideal value is 1 , values close to 1 or -1 are equally acceptable since the machine-learning algorithm while optimising for $f\left(I_{m}, J_{n}\right)$ can simply multiply coefficients with +1 or -1 to align the hypothesis with the actual heat-flux. However, values close to 0 indicate the hypothesis vector is perpendicular to the heat-flux and no coefficient will be able to change this orthogonal behaviour. Looking at Fig. 12, it is evident from the near wall region that the SGDH has values in the range of $[0,0.2]$ whereas the GGDH can achieve values in the range [0.8,1]. It is important to emphasise that the alignment is a powerful a priori metric for assessing a model's eventual performance $a$ posteriori with a host of published studies demonstrating this $[10,12,15,28]$. Thus, building a machine-learnt closure and conducting calculations with the GGDH should help realise further improvements, while being generalisable, and thus will be part of future investigations.

\section{CONCLUSION}

In this study, we conducted unsteady RANS calculations for a fundamental trailing-edge slot configuration to assess the predictive performance of the adiabatic wall-effectiveness $\eta$, when compared with highly resolved LES. To this end, closures for the turbulence stress and the heat-flux were developed using a novel machine-learning algorithm that produces equations for these closures. Importantly, the closures for both quantities were developed only for the stochastic length scales by first processing the reference LES dataset. This exercise was conducted only for the $\mathrm{BR}=1.26$ case, since it was observed that the closure for the turbulence stress was re-usable on the other BRs as well with good performance $a$ priori. Calculations with only the closure for the turbulence stress showed a significant improvement in predicting $\eta$ when compared with a baseline URANS without any machine-learnt closures for $\mathrm{BR}=1.26$. This improvement was attributed to the non-linear terms in the closure, which were responsible for resolving the correct amount of mixing at the near slot exit. Similar levels of improvement were observed when the same closure was applied to the $\mathrm{BR}=0.86$ and 1.07 cases, confirming the a priori conclusions regarding this closure. In the next stage, the machine-learnt heat-flux closure for $1 / \mathrm{Pr}_{t}$ was implemented and coupled calculations were conducted where additional improvements were seen over the case using a trained stress closure with the baseline heat-flux closure for the BR = 1.26 case. However, running the coupled closure calculations on the remaining BRs showed a poorer prediction than when using only the trained stress closure and it was concluded that the heatflux closure was more sensitive to the flow conditions, which was found by generating heat-flux closures for $B R=0.86$ and 1.07. The limited improvement and lack of generalisation was attributed to the use of the standard gradient diffusion hypothesis for the heat-flux closure, since the temperature gradient vector was found to be orthogonal to the heat-flux vector, especially near the wall. Thus, to realise further gains in the prediction of $\eta$ in a generalisable sense, it is recommended to develop heatflux closures using the generalised gradient diffusion hypothesis instead, since the a priori analysis shows a better alignment between the heat-flux vector and the resultant hypothesis vector. 


\section{ACKNOWLEDGMENT}

This work was supported by resources provided by the Pawsey Supercomputing Centre with funding from the Australian Government and the Government of Western Australia. This work was also financially supported by the University of Melbourne PhD Write-up Grant.

\section{REFERENCES}

[1] Kacker, S. C., and Whitelaw, J. H., 1969. "An experimental investigation of the influence of slot-lip-thickness on the impervious-wall effectiveness of the uniform-density, twodimensional wall jet”. Int. J. Heat Mass Transf., 12(9), pp. 1196-1201.

[2] Kacker, S. C., and Whitelaw, J. H., 1971. "The turbulence characteristics of two-dimensional wall-jet and wall-wake flows". J. Appl. Mech., 38(1), pp. 239-252.

[3] Joo, J., and Durbin, P. A., 2009. "Simulation of Turbine Blade Trailing Edge Cooling". J. Fluids Eng., 131(2), p. 021102.

[4] Ivanova, E., and Laskowski, G., 2014. "LES and Hybrid RANS/LES of a Fundamental Trailing Edge Slot". In Turbomach. Tech. Conf. Expo. GT2014-25906, pp. 1-9.

[5] Launder, B. E., and Rodi, W., 1983. "The Turbulent Wall Jet Measurements and Modeling”. Annu. Rev. Fluid Mech., 15(1), pp. 429-459.

[6] Holloway, D. S., Leylek, J. H., and Buck, F. A., 2002. "Pressure-side Bleed Film Cooling: Part I - Steady Framework for Experimental and Computational Results". In Turbomach. Tech. Conf. Expo. GT2002-30471, ASME, pp. 1-9.

[7] Holloway, D. S., Leylek, J. H., and Buck, F. A., 2002. "Pressure-side Bleed Film Cooling: Part II - Unsteady Framework for Experimental and Computational Results". In Turbomach. Tech. Conf. Expo. GT2002-30472, ASME, pp. 1-9.

[8] Effendy, M., Yao, Y. F., Yao, J., and Marchant, D. R., 2016. "DES study of blade trailing edge cutback cooling performance with various lip thicknesses". Appl. Therm. Eng., 99, pp. 434-445.

[9] Schneider, H., Von Terzi, D. A., Bauer, H. J., and Rodi, W., 2015. "Coherent structures in trailing-edge cooling and the challenge for turbulent heat transfer modelling". Int. J. Heat Fluid Flow, 51, pp. 110-119.

[10] Sandberg, R. D., Tan, R., Weatheritt, J., Ooi, A., Haghiri, A., Michelassi, V., and Laskowski, G., 2018. "Applying Machine Learnt Explicit Algebraic Stress and Scalar Flux Models To a Fundamental Trailing Edge Slot”. J. Turbomach., 140(October), pp. 1-13.

[11] Milani, P. M., Ling, J., and Eaton, J. K., 2018. "Physical Interpretation of Machine Learning Models Applied to Film Cooling Flows". ASME Turbo Expo, 141(51081), p. V05AT12A019.

[12] Weatheritt, J., and Sandberg, R. D., 2016. "A novel evolutionary algorithm applied to algebraic modifications of the RANS stress-strain relationship". J. Comput. Phys., 325, pp. 22-37.

[13] Weatheritt, J., Sandberg, R. D., Ling, J., Saez, G., and Bodart, J., 2017. "A Comparative Study of Contrasting Ma- chine Learning Frameworks Applied to RANS Modeling of Jets in Crossflow". In Turbomach. Tech. Conf. Expo. GT2017-63403, pp. 1-12.

[14] Lav, C., Philip, J., and Sandberg, R. D., 2019. "A New Data-Driven Turbulence Model Framework for Unsteady Flows Applied to Wall-Jet and Wall-Wake Flows". In ASME Turbo Expo 2019 GT2019-90179, ASME, pp. 1-12.

[15] Lav, C., Sandberg, R. D., and Philip, J., 2019. "A Framework to Develop Data-Driven Turbulence Models for Flows with Organised Unsteadiness". J. Comput. Phys., 383, feb, pp. 148-165.

[16] Menter, F. R., Kuntz, M., and Langtry, R., 2003. “Ten years of industrial experience with the SST turbulence model". Turbul. Heat Mass Transf., 4(1), pp. 625-632.

[17] Schmitt, F. G., 2007. “About Boussinesq's turbulent viscosity hypothesis: historical remarks and a direct evaluation of its validity". Comptes Rendus - Mec., 335(9-10), pp. 617-627.

[18] Pope, S. B., 1975. "A more general effective-viscosity hypothesis". J. Fluid Mech., 72(2), pp. 331-340.

[19] Wallin, S., and Johansson, A. V., 2000. "An explicit algebraic Reynolds stress model for incompressible and compressible turbulent flows". J. Fluid Mech., 403, pp. 89-132.

[20] Daly, B. J., and Harlow, F. H., 1970. "Transport equations in turbulence". Phys. Fluids, 13(11), pp. 2634-2649.

[21] Hussain, A. K. M. F., and Reynolds, W. C., 1970. "The mechanics of an organized wave in turbulent shear flow". J. Fluid Mech., 41(02), pp. 241-258.

[22] Nicoud, F., and Ducros, F., 1999. "Subgrid-Scale Stress Modelling Based on the Square of the Velocity Gradient Tensor". Flow, Turbul. Combust., 62, pp. 183-200.

[23] Sandberg, R. D., Michelassi, V., Pichler, R., Chen, L.-w., and Johnstone, R., 2015. "Compressible direct numerical simulation of low-pressure turbines: part I - methodology". J. Turbomach., 137(5), pp. 051011-1-10.

[24] Kacker, S. C., and Whitelaw, J. H., 1968. "Some properties of the two-dimensional, turbulent wall jet in a moving stream”. J. Appl. Mech., 35, pp. 641-651.

[25] Eucken, A., 1913. "Über das Wärmeleitvermögen, die spezifische Wärme und die innere Reibung der Gase". Phys. Z, 14(8), pp. 324-332.

[26] Milani, P. M., Ling, J., Saez-Mischlich, G., Bodart, J., and Eaton, J. K., 2018. "A machine learning approach for determining the turbulent diffusivity in film cooling flows". $J$. Turbomach., 140(2), pp. 1-8.

[27] Hussain, A. K. M. F., 1983. "Coherent structures - reality and myth". Phys. Fluids, 26(10), p. 2816.

[28] Weatheritt, J., Pichler, R., Sandberg, R. D., Laskowski, G., and Michelassi, V., 2017. "Machine Learning For Turbulence Model Development Using A High-Fidelity HPT Cascase Simulation". In Turbomach. Tech. Conf. Expo. GT2017-63497, pp. 1-12. 


\section{University Library}

\section{- M M N E R VA A gateway to Melbourne's research publications}

Minerva Access is the Institutional Repository of The University of Melbourne

Author/s:

Lav, C;Sandberg, R

Title:

UNSTEADY SIMULATIONS OF A TRAILING-EDGE SLOT USING MACHINE-LEARNT TURBULENCE STRESS AND HEAT-FLUX CLOSURES

Date:

2020-06-22

\section{Citation:}

Lav, C. \& Sandberg, R. (2020). UNSTEADY SIMULATIONS OF A TRAILING-EDGE SLOT USING MACHINE-LEARNT TURBULENCE STRESS AND HEAT-FLUX CLOSURES.

Proceedings of ASME Turbo Expo 2020: Turbomachinery Technical Conference \& Exposition GT2020, 7B-2020, ASME: The American Society of Mechanical Engineers. https:// doi.org/10.1115/GT2020-14398.

Persistent Link:

http://hdl.handle.net/11343/241910 